\title{
4th Workshop - Insulin Resistance. Early Diagnosis? Early Treatment? - Keynote Speaker
}

\section{Adipokines and insulin resistance: interaction with physical activity and fitness}

\author{
Sonia Gomez-Martinez, Ligia-Esperanza Diaz, Fatima Perez de Heredia, David Martinez- \\ Gomez and Ascension Marcos
}

Immunonutrition Research Group, Department of Metabolism and Nutrition, Institute of Food Science, Technology and Nutrition (ICTAN) (former Instituto Frío), Spanish National Research Council (CSIC), Madrid, Spain

Adipose tissue-derived proteins, or 'adipokines', exert a wide range of physiological actions, connecting this tissue with the rest of organs and systems in the body, and thus linking fat storage depots with central regulation of energy balance, metabolic homeostasis, immune status, reproductive capacity, angiogenesis or inflammatory response (Trayhurn and Wood 2004). Adipose tissue dysfunction in obesity takes place with an altered production of most adipokines; this outcome is also shown in related pathological conditions such as type 2 diabetes mellitus and the metabolic syndrome.

Lifestyle habits, in particular physical activity, are modifiable risk factors for obesity and obesity-related disturbances. Both physical activity and fitness have been inversely associated with all-cause mortality and chronic diseases, including CVD, in early ages as well as in adulthood
(Eisenmann 2007; Kamper et al. 1996). Adipose tissuederived proteins, or adipokines, have revealed themselves as key modulators of energy homeostasis and inflammatory balance, playing a determinant role in the regulation of systemic insulin sensitivity.

The mechanisms through which physical activity and fitness influence chronic disease risk have not been entirely clarified yet, although the new inflammatory markers, amongst them adipokines, are likely candidates in mediating physical activity and fitness health benefits. Physical activity and physical fitness provide many health benefits, which appear to be not only a consequence of changes in body weight and composition, but that may also be mediated by stimulation of insulin sensitivity and amelioration of the inflammatory response, through direct and indirect regulatory actions on adipokine secretion.

\section{What are the roles of insulin and other hormones in the development of obesity and its complications in children?}

\author{
Éva Erhardt
}

Head of the Division of Endocrinology and Diabetology at the Department of Paediatrics, University of Pecs, Pecs, Hungary

Introduction: The epidemic of childhood obesity is associated with the occurrence of comorbidities including type 2 diabetes mellitus (T2DM), hypertension, metabolic syndrome, non-alcoholic fatty liver disease, polycystic ovary syndrome and others. Excess body fat may be stored by increasing the size (hypertrophy) and/or the number (hyperplasia) of adipocytes. White adipose tissue has been recognized as an endocrine organ that secretes a number of hormones and cytokines (adipokines: leptin, adiponectin, resistin, visfatin), which interact with brain centres and play a role in the pathogenesis of obesity-associated diseases. Obesity is the most common cause of insulin resistance. Insulin is also an important regulator of lipolysis and lipogenesis.

Examination: Oral glucose tolerance test was performed in 289 obese (mean BMI 31.1 (SD $4 \cdot 6$ ) $\mathrm{kg} / \mathrm{m}^{2}$ ) adolescents (mean age 12.9 (SD $2 \cdot 7$ ) years), among them the impaired glucose tolerance (IGT) was found in fifty (17.3\%) and T2DM in five $(1.9 \%)$ children. After a 6 months lifestyle and dietary changes the metabolic status of obese children with IGT and T2DM could improve (Table 1).

Conclusions: Childhood obesity is frequently associated with the disturbances of carbohydrate metabolism. 